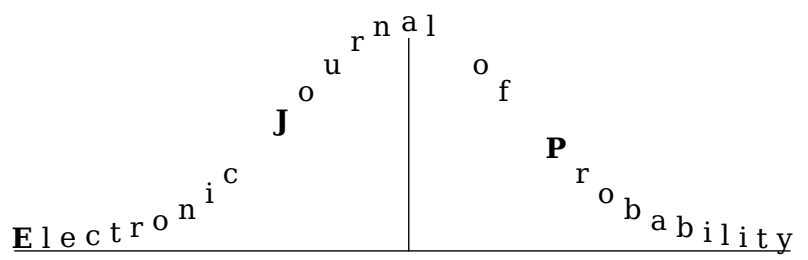

Electron. J. Probab. 24 (2019), no. 107, 1-15.

ISSN: 1083-6489 https://doi.org/10.1214/19-EJP365

\title{
Estimates of norms of log-concave random matrices with dependent entries*
}

\author{
Marta Strzelecka ${ }^{\dagger}$
}

\begin{abstract}
We prove estimates for $\mathbb{E}\left\|X: \ell_{p^{\prime}}^{n} \rightarrow \ell_{q}^{m}\right\|$ for $p, q \geqslant 2$ and any random matrix $X$ having the entries of the form $a_{i j} Y_{i j}$, where $Y=\left(Y_{i j}\right)_{1 \leqslant i \leqslant m, 1 \leqslant j \leqslant n}$ has i.i.d. isotropic log-concave rows and $p^{\prime}$ denotes the Hölder conjugate of $p$. This generalises a result of Guédon, Hinrichs, Litvak, and Prochno for Gaussian matrices with independent entries. Our estimate is optimal up to logarithmic factors. As a byproduct we provide an analogous bound for $m \times n$ random matrices, whose entries form an unconditional vector in $\mathbb{R}^{m n}$. We also prove bounds for norms of matrices whose entries are certain Gaussian mixtures.
\end{abstract}

Keywords: random matrices; operator norm; log-concave vectors; unconditional vectors. AMS MSC 2010: 60B20; 46B09; 15B52.

Submitted to EJP on April 3, 2019, final version accepted on September 18, 2019.

\section{Introduction and main results}

By $\|A\|_{p, q}$ we denote the operator norm of the matrix $A$ from $\ell_{p}$ to $\ell_{q}$. A classical result regarding spectra of random matrices is Wigner's Semicircle Law, which describes the limit of the empirical spectral measure of a random matrix with independent centred entries with equal variance. Theorems of this type say nothing about the largest eigenvalue (i.e. the operator norm). However, Seginer proved in [16] that for a random matrix $X$ with i.i.d. symmetric entries $\mathbb{E}\|X\|_{2,2}$ is of the same order as the expectation of the maximum Euclidean norm of rows and columns of $X$. The same holds true for structured Gaussian matrices (i.e. when $X_{i j}=a_{i j} g_{i j}$ and $g_{i j}$ are i.i.d. standard Gaussian variables), as was shown recently by Latała, van Handel, and Youssef in [14], and up to a logarithmic factor for any random matrix $X$ with independent centred entries, see [15]. The main novelty of these two results is that they do not require the entries of $X$ to be equally distributed (nor to have equal variances).

\footnotetext{
*The research was supported by the National Science Centre, Poland via the grants 2015/19/N/ST1/02661 and 2018/28/T/ST1/00001.

${ }^{\dagger}$ Institute of Mathematics, University of Warsaw, Poland. E-mail: martast@mimuw.edu.pl
} 
In [9] another upper bound for $\mathbb{E}\|X\|_{2,2}$ was proved:

$$
\mathbb{E}\|X\|_{2,2} \leqslant C\left[\max _{i}\left(\sum_{j} \mathbb{E} X_{i j}^{2}\right)^{1 / 2}+\max _{j}\left(\sum_{i} \mathbb{E} X_{i j}^{2}\right)^{1 / 2}+\left(\sum_{i, j} \mathbb{E} X_{i j}^{4}\right)^{1 / 4}\right],
$$

where $C$ is a universal constant. It also requires the independence of entries, but not the equality of their distributions. This bound is dimension free, but is in some cases worse than the one from [15].

Upper bounds for the expectation of other operator norms were investigated in [2] in the case of independent centred entries bounded by 1 . For $q \geqslant 2$ and $m \times n$ matrices the authors proved that $\mathbb{E}\|X\|_{2, q} \lesssim \max \left\{m^{1 / q}, \sqrt{n}\right\}$. In [6] Guédon, Hinrichs, Litvak, and Prochno proved that for a structured Gaussian matrix $X=\left(a_{i j} X_{i j}\right)_{i \leqslant m, j \leqslant n}$ and $p, q \geqslant 2$,

$$
\begin{aligned}
\mathbb{E}\|X\|_{p^{\prime}, q} \leqslant C(p, q)\left[(\log m)^{1 / q} \max _{1 \leqslant i \leqslant m}\left(\sum_{j=1}^{n}\left|a_{i j}\right|^{p}\right)^{1 / p}\right. & +\max _{1 \leqslant j \leqslant n}\left(\sum_{i=1}^{m}\left|a_{i j}\right|^{q}\right)^{1 / q} \\
& \left.+(\log m)^{1 / q} \mathbb{E} \max _{\substack{1 \leqslant i \leqslant m \\
1 \leqslant j \leqslant n}}\left|X_{i j}\right|\right] .
\end{aligned}
$$

This estimate is optimal up to logarithmic factors (see Remark 1.2 below). Estimating more general norms required a different approach than those used for estimating the spectral norm. In particular the moment method fails in estimating $\mathbb{E}\|X\|_{p^{\prime}, q}$ for $(p, q) \neq(2,2)$ as it gives information only about the spectrum of $X$.

All the results mentioned above require the independence of entries of $X$. We will show how to relax the independence assumption. The aim of this article is to generalise the main result of [6] to a wide class of random matrices with independent uncorrelated log-concave rows by following the scheme of proof of the original theorem from [6]. We work with a more general class of random matrices, thus the proof from [6] may not be rewritten verbatim, but it requires some extra tools: the comparison of weak and strong moments of $\ell_{p}$-norm of $X$ from [11] and a Sudakov minoration-type bound from [10].

Before we state our main results, let us say a few words about log-concave vectors, which are widely investigated in convex geometry and high dimensional probability. We call a random vector $Z$ in $\mathbb{R}^{n}$ log-concave, if for any compact nonempty sets $K, L \subset \mathbb{R}^{n}$ and $\lambda \in[0,1]$,

$$
\mathbb{P}(Z \in \lambda K+(1-\lambda) L) \geqslant \mathbb{P}(Z \in K)^{\lambda} \mathbb{P}(Z \in L)^{1-\lambda} .
$$

The class of log-concave vectors is closed under linear transformations, convolutions and weak limits. By the result of Borell [3] an $n$-dimensional vector with a full dimensional support is log-concave if and only if it has a log-concave density, i.e. a density (with respect to the Lebesgue measure) of the form $e^{-h}$, where $h$ is a convex function with values in $(-\infty, \infty]$.

Log-concave vectors are a natural generalisation of vectors distributed uniformly over convex bodies. The distribution of any log-concave vector can be obtained as a weak limit of projections of uniform measures over (higher dimensional) convex bodies (see for example [1]).

We will frequently use a basic property of log-concave vectors: the regularity of $f(Z)$ for log-concave vectors $X$ and seminorms $f$, which states that

$$
\left(\mathbb{E} f(Z)^{p}\right)^{1 / p} \leqslant C_{1} \frac{p}{q}\left(\mathbb{E} f(Z)^{q}\right)^{1 / q} \text { for } p \geqslant q \geqslant 1,
$$

where $C_{1}$ is a universal constant (see [4, Theorem 2.4.6]). Other results and conjectures concerning log-concave vectors are discussed in the monograph [4]. 
We say that a vector in $\mathbb{R}^{n}$ is isotropic if its covariance matrix is the identity. If $Z$ is a log-concave random vector in $\mathbb{R}^{n}$ with a full dimensional support, then there exists a linear transformation $T$ such that $\operatorname{Cov}(T Z)=\mathrm{Id}$, so the isotropicity is only a matter of normalisation.

If $A=\left(A_{i j}\right)_{i \leqslant m, j \leqslant n}$ is an $m \times n$ matrix, we denote by $A_{i} \in \mathbb{R}^{n}$ its $i$-th row and by $A^{(j)} \in \mathbb{R}^{m}$ its $j$-th column. We are now ready to present the main theorem.

Theorem 1.1. Let $m \geqslant 2$, let $Y_{1}, \ldots, Y_{m}$ be i.i.d. isotropic log-concave vectors in $\mathbb{R}^{n}$, and let $A=\left(A_{i j}\right)$ be an $m \times n$ (deterministic) matrix. Consider a random matrix $X$ with entries $X_{i j}=A_{i j} Y_{i j}$ for $i \leqslant m, j \leqslant n$, where $Y_{i j}$ is the $j$-th coordinate of $Y_{i}$. Then for every $p, q \geqslant 2$ we have

$$
\mathbb{E}\|X\|_{p^{\prime}, q} \leqslant C(p, q)\left[(\log m)^{1 / q} \max _{1 \leqslant i \leqslant m}\left\|A_{i}\right\|_{p}+\max _{1 \leqslant j \leqslant n}\left\|A^{(j)}\right\|_{q}+(\log m)^{1+\frac{1}{q}} \mathbb{E} \max _{\substack{1 \leqslant i \leqslant m \\ 1 \leqslant j \leqslant n}}\left|X_{i j}\right|\right],
$$

where $C(p, q)$ depends only on $p$ and $q$.

Let us stress that the theorem requires the independence only between the rows and does not require the independence of the entries of $X$.

Remark 1.2. Note that the bound from Theorem 1.1 is optimal up to a constant depending on $p, q$ and logarithmically on the dimension. Indeed, since $Y_{i j}$ is log-concave we have by the regularity of $Y_{i j}$ (see (1.2)) that $\mathbb{E}\left|Y_{i j}\right| \geqslant\left(2 C_{1}\right)^{-1}\left(\mathbb{E} Y_{i j}^{2}\right)^{1 / 2}=\left(2 C_{1}\right)^{-1}$. Hence for every $j \leqslant n$,

$$
\mathbb{E}\|X\|_{p^{\prime}, q}=\mathbb{E} \sup _{u \in \ell_{p^{\prime}}^{n}}\|X u\|_{q} \geqslant \mathbb{E}\left\|X e_{j}\right\|_{q}=\mathbb{E}\left\|X^{(j)}\right\|_{q}=\mathbb{E}\left\|\left(\left|Y_{i j}\right| A_{i j}\right)_{i}\right\|_{q} \geqslant\left(2 C_{1}\right)^{-1}\left\|A^{(j)}\right\|_{q} .
$$

Since $\|X\|_{p^{\prime}, q}=\left\|X^{T}\right\|_{q^{\prime}, p}$, we also have $\mathbb{E}\|X\|_{p^{\prime}, q} \geqslant\left(2 C_{1}\right)^{-1}\left\|A_{i}\right\|_{p}$ for all $i \leqslant m$. Moreover, for all $i \leqslant m$ and $j \leqslant n$,

$$
\|X\|_{p^{\prime}, q}=\sup _{u \in \ell_{p^{\prime}}^{n}} \sup _{v \in \ell_{q^{\prime}}^{n}} v^{T} X u \geqslant e_{i}^{T} X\left(\operatorname{sgn} X_{i j} e_{j}\right)=\left|X_{i j}\right| .
$$

Therefore

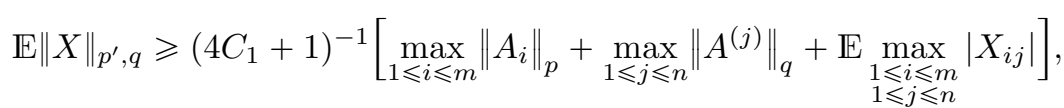

which yields the claim.

Note that in (1.3) the logarithmic term appears in front of the norm of rows, but not in front of the norm of columns, so our bound is not symmetric. This is not so strange, since the assumptions of the theorem are also non-symmetric: we assume that the rows are weighted i.i.d. random vectors, but no independence between the columns is required. However, the asymmetry of the bound in Theorem 1.1 is mainly a residue of the proof and the author does not know if one may skip the logarithmic factor in front of the norm of rows.

Since $\|X\|_{p^{\prime}, q}=\left\|X^{T}\right\|_{q^{\prime}, p}$, one can assume in (1.3) that the columns (instead of the rows) of $Y$ are i.i.d. isotropic log-concave vectors. Then Theorem 1.1 yields

$$
\mathbb{E}\|X\|_{p^{\prime}, q} \leqslant C(p, q)\left[\max _{1 \leqslant i \leqslant m}\left\|A_{i}\right\|_{p}+(\log n)^{1 / p} \max _{1 \leqslant j \leqslant n}\left\|A^{(j)}\right\|_{q}+(\log n)^{1+\frac{1}{p}} \mathbb{E} \max _{\substack{1 \leqslant i \leqslant m \\ 1 \leqslant j \leqslant n}}\left|X_{i j}\right|\right] .
$$

We mentioned previously results from $[16,14,15]$, which provide the bounds of the expected value of the operator norm in terms of expected values of norms of rows 
Estimates of norms of log-concave random matrices with dependent entries

and columns. Theorem 1.1 easily implies an estimate of the same kind: by (1.2), the assumption $\mathbb{E} Y_{i j}^{2}=1$, and the Jensen inequality we get

$$
\begin{gathered}
(\log m)^{1 / q} \max _{1 \leqslant i \leqslant m}\left\|A_{i}\right\|_{p}+\max _{1 \leqslant j \leqslant n}\left\|A^{(j)}\right\|_{q}+(\log m)^{1+\frac{1}{q}} \mathbb{E} \max _{\substack{1 \leqslant i \leqslant m \\
1 \leqslant j \leqslant n}}\left|X_{i j}\right| \\
\leqslant 2 C_{1}\left((\log m)^{1 / q} \max _{1 \leqslant i \leqslant m}\left\|\left(A_{i j} \mathbb{E}\left|Y_{i j}\right|\right)_{j}\right\|_{p}+\max _{1 \leqslant j \leqslant n}\left\|\left(A_{i j} \mathbb{E}\left|Y_{i j}\right|\right)_{i} \mid\right\|_{q}\right. \\
\left.+(\log m)^{1+\frac{1}{q}} \mathbb{E} \max _{\substack{1 \leqslant i \leqslant m \\
1 \leqslant j \leqslant n}}\left|X_{i j}\right|\right) \\
\leqslant 2 C_{1}\left((\log m)^{1 / q} \mathbb{E} \max _{1 \leqslant i \leqslant m}\left\|X_{i}\right\|_{p}+\mathbb{E} \max _{1 \leqslant j \leqslant n}\left\|X^{(j)}\right\|_{q}+(\log m)^{1+\frac{1}{q}} \mathbb{E} \max _{1 \leqslant i \leqslant m}\left\|X_{i}\right\|_{p}\right) \\
\leqslant C\left((\log m)^{1+\frac{1}{q}} \mathbb{E} \max _{1 \leqslant i \leqslant m}\left\|X_{i}\right\|_{p}+\mathbb{E} \max _{1 \leqslant j \leqslant n}\left\|X^{(j)}\right\|_{q}\right),
\end{gathered}
$$

where $C$ is a universal constant. Therefore (1.3) yields the following corollary.

Corollary 1.3. Under the assumptions of Theorem 1.1 we have

$$
\mathbb{E}\|X\|_{p^{\prime}, q} \leqslant C(p, q)\left((\log m)^{1+\frac{1}{q}} \mathbb{E} \max _{1 \leqslant i \leqslant m}\left\|X_{i}\right\|_{p}+\mathbb{E} \max _{1 \leqslant j \leqslant n}\left\|X^{(j)}\right\|_{q}\right) .
$$

Remark 1.4. If the rows and columns of a random matrix $Y$ are isotropic and log-concave (we do not require independence), and $p, q \geqslant 1$, then

$$
\begin{aligned}
& \mathbb{E} \max _{1 \leqslant i \leqslant m}\left(\sum_{j=1}^{n}\left|A_{i j} Y_{i j}\right|^{p}\right)^{1 / p}+\mathbb{E} \max _{1 \leqslant j \leqslant n}\left(\sum_{i=1}^{m}\left|A_{i j} Y_{i j}\right|^{q}\right)^{1 / q} \\
& \quad \leqslant C\left(p^{2} \max _{1 \leqslant i \leqslant m}\left\|A_{i}\right\|_{p}+q^{2} \max _{1 \leqslant j \leqslant n}\left\|A^{(j)}\right\|_{q}+(p+q) \log (m \vee n) \mathbb{E} \max _{\substack{1 \leqslant i \leqslant m \\
1 \leqslant j \leqslant n}}\left|A_{i j} Y_{i j}\right|\right) .
\end{aligned}
$$

This means that inequality (1.4) may be reversed up to a logarithmic factor and constants depending only on $p$ and $q$ in the log-concave setting. Therefore the estimates from Theorem 1.1 and Corollary 1.3 are equivalent up to a logarithmic factor.

Inequality (1.5) follows directly from the following proposition.

Proposition 1.5. Let $Y$ be an $m \times n$ random matrix, with isotropic and log-concave rows, let $B$ be a deterministic $m \times n$ matrix, and let $p \geqslant 1$. Then

$$
\mathbb{E} \max _{1 \leqslant i \leqslant m}\left(\sum_{j=1}^{n}\left|B_{i j} Y_{i j}\right|^{p}\right)^{1 / p} \leqslant C\left(p^{2} \max _{1 \leqslant i \leqslant m}\left(\sum_{j=1}^{n}\left|B_{i j}\right|^{p}\right)^{1 / p}+p \log (m \vee n) \mathbb{E} \max _{\substack{1 \leqslant i \leqslant m \\ 1 \leqslant j \leqslant n}}\left|B_{i j} Y_{i j}\right|\right),
$$

where $C$ is a universal constant.

Our next result concerns unconditional matrices. Recall that we say that a random vector $Z$ in $\mathbb{R}^{d}$ is unconditional, if for every choice of signs $\eta \in\{-1,1\}^{d}$ the vectors $Z$ and $\left(\eta_{i} Z_{i}\right)_{i \leqslant d}$ are equally distributed (or, equivalently, that $Z$ and $\left(\varepsilon_{i} Z_{i}\right)_{i \leqslant d}$ are equally distributed, where $\varepsilon_{1}, \ldots, \varepsilon_{d}$ are i.i.d. symmetric Bernoulli variables, independent of $Z$ ). The assertion of the next corollary is expressed in the spirit of Corollary 1.3, which is more natural in the non log-concave setting (without the assumption of log-concavity inequality (1.5) may no longer be true, even up to additional logarithmic factors).

Corollary 1.6. Assume that $X$ is a random matrix such that the $(m n)$-dimensional vector $\left(X_{1,1}, \ldots X_{1, n}, X_{2,1}, \ldots, X_{2, n}, X_{m, 1}, \ldots, X_{m n}\right)$ is unconditional. Then for every $p, q \geqslant 2$ we have

$$
\mathbb{E}\|X\|_{p^{\prime}, q} \leqslant C(p, q)\left((\log m)^{\frac{1}{2}+\frac{1}{q}} \mathbb{E} \max _{1 \leqslant i \leqslant m}\left(\sum_{j=1}^{n}\left|X_{i j}\right|^{p}\right)^{1 / p}+\sqrt{\log n} \mathbb{E} \max _{1 \leqslant j \leqslant n}\left(\sum_{i=1}^{m}\left|X_{i j}\right|^{q}\right)^{1 / q}\right),
$$

where $C(p, q)$ depends only on $p$ and $q$. 
The asymmetry of the bound in (1.6) is just a residue of its proof: the factor $(\log m)^{1 / q}$ could be skipped if one could prove (1.1) in the Gaussian case with constants independent of the dimensions. Such an improved version of (1.1) was conjectured in [6].

Let us compare (1.6) with the following result of Seginer coming from [16]. Let $X=\left(A_{i j} \varepsilon_{i j}\right)_{1 \leqslant i \leqslant m, 1 \leqslant j \leqslant n}$ where $\varepsilon_{i j}$ are independent random signs. Then

$$
\mathbb{E}\|X\|_{2 \rightarrow 2} \leqslant C \sqrt[4]{\log \min (m, n)}\left(\max _{1 \leqslant i \leqslant m}\left\|A_{i}\right\|_{2}+\max _{1 \leqslant j \leqslant n}\left\|A^{(j)}\right\|_{2}\right),
$$

where $C$ is a universal constant. Moreover, for every $m, n$, there exists a matrix $\left(A_{i j}\right)_{1 \leqslant i \leqslant m, 1 \leqslant j \leqslant n}$, for which (1.7) may be reversed (up to a universal constant). Therefore one may not skip the dependence on the dimension also in estimate (1.6).

In the case $(p, q)=(2,2)$ and $X=\left(A_{i j} \varepsilon_{i j}\right)$ Corollary 1.6 provides a bound with worse dependence of the dimension than in (1.7). However, our result works also in much more general setting than the result of Seginer does (in particular, our Corollary works also when the entries of $X$ are dependent).

The rest of this note is organised as follows. Section 2 contains results from other articles to be used in a sequel. Section 3 contains generalisations of Lemmas 3.1 and 3.2 from [6] to the log-concave setting and the proof of Theorem 1.1. In Section 4 we show how to deduce an analogue of Theorem 1.1 for Gaussian mixtures (see Corollary 4.2) and we provide a proof of Proposition 1.5. Section 5 is devoted to the proof of Corollary 1.6 .

Notation By $C$ we denote universal constants. If a constant $C$ depends on a parameter $\alpha$, we express it as $C(\alpha)$. The value of $C, C(\alpha)$ may differ at each occurrence. Whenever we want to fix the value of an absolute constant we use letters $C_{1}, C_{2}, \ldots$ We may and do always assume that $C, C_{i} \geqslant 1$. For two quantities $a, b$ we write $a \lesssim b$ if there exists a constant $C$, such that $a \leqslant C b$, and $a \sim b$, if $a \lesssim b$ and $b \lesssim a$. For two numbers $a$ and $b$ we write $a \vee b$ instead of $\max \{a, b\}$.

For a random variable $Z$ by $\|Z\|_{p}$ we denote the $p$-th integral norm of $Z$, i.e. the quantity $\left(\mathbb{E}|Z|^{p}\right)^{1 / p}$; in the case when $Z=\|U\|$ we also call this quantity the $p$-th strong moment of a random vector $U$ associated with the norm $\|\cdot\|$. For a vector $x \in \mathbb{R}^{n}$ (in particular for a random vector $Z$ ) and $r \geqslant 1$, by $\|x\|_{r}$ we denote the $\ell_{r}$-norm of $x$, i.e. $\|x\|_{r}:=\left(\sum_{i=1}^{n}\left|x_{i}\right|^{r}\right)^{1 / r}$. For $r=2$ we shall also write $|\cdot|$ instead of $\|\cdot\|_{2}$. It will be always clear from the context, what $\|Z\|_{q}$ means for a random object $Z$, so the double meaning of $\|\cdot\|_{q}$ will not lead to any misunderstanding. Recall that for an $m \times n$ matrix $A$ by $\|A\|_{p, q}$ we denote its norm from $\ell_{p}^{n}$ to $\ell_{q}^{m}$. For $p \in[1, \infty]$ we denote by $p^{\prime}$ the Hölder conjugate of $p$, i.e. the number such that $1=\frac{1}{p}+\frac{1}{p^{\prime}}$.

\section{Preliminaries}

In the proof of the main theorem we will need the comparison of weak and strong moments for $\ell_{p}$-norms of log-concave vectors:

Theorem 2.1 ([11, Theorem 5]). Let $Z$ be a log-concave vector in $\mathbb{R}^{n}$ and let $p \in[1, \infty)$. Then

$$
\left(\mathbb{E}\|Z\|_{p}^{q}\right)^{1 / q} \leqslant C p\left(\mathbb{E}\|Z\|_{p}+\sigma_{p, Z}(q)\right) \quad \text { for } q \geqslant 1
$$

where

$$
\sigma_{p, Z}(q):=\sup _{t \in B_{p^{\prime}}^{n}}\left\|\sum_{i=1}^{n} t_{i} Z_{i}\right\|_{q}
$$

is the $q$-th weak moment of $X$ associated with the $\ell_{p}$-norm. 
Estimates of norms of log-concave random matrices with dependent entries

We will use the previous theorem also in the tail-bound version:

Corollary 2.2. Assume $Z$ is a log-concave vector in $\mathbb{R}^{n}$ and $p \in[1, \infty)$. Then for all $u>0$,

$$
\mathbb{P}\left(\|Z\|_{p} \geqslant C_{2} p\left(u+\mathbb{E}\|Z\|_{p}\right)\right) \leqslant C_{3} \sup _{t \in B_{p^{\prime}}^{n}} \mathbb{P}\left(\left|\sum_{i=1}^{n} t_{i} Z_{i}\right| \geqslant u\right) .
$$

For the Reader's convenience we give a proof of this corollary, which goes along the lines of the proof of Corollary 1.3 in [12].

Proof. Define a random variable $S:=\|Z\|_{p}$. By the Paley-Zygmund inequality and (1.2) we have for $t \in \mathbb{R}^{n}$, and $q \geqslant 1$,

$$
\begin{aligned}
\mathbb{P}\left(\left|\sum_{i=1}^{n} t_{i} Z_{i}\right| \geqslant \frac{1}{2}\left\|\sum_{i=1}^{n} t_{i} Z_{i}\right\|_{q}\right) & =\mathbb{P}\left(\left|\sum_{i=1}^{n} t_{i} Z_{i}\right|^{q} \geqslant 2^{-q} \mathbb{E}\left|\sum_{i=1}^{n} t_{i} Z_{i}\right|^{q}\right) \\
& \geqslant\left(1-2^{-q}\right)^{2}\left(\frac{\left\|\sum_{i=1}^{n} t_{i} Z_{i}\right\|_{q}}{\left\|\sum_{i=1}^{n} t_{i} Z_{i}\right\|_{2 q}}\right)^{2 q} \geqslant e^{-C_{4} q}
\end{aligned}
$$

In order to show (2.1) we consider 3 cases.

Case 1. $2 u<\sup _{t \in B_{p^{\prime}}^{n}}\left\|\sum_{i=1}^{n} t_{i} Z_{i}\right\|_{2}$. Then by (2.2)

$$
\sup _{t \in B_{p^{\prime}}^{n}} \mathbb{P}\left(\left|\sum_{i=1}^{n} t_{i} Z_{i}\right| \geqslant u\right) \geqslant e^{-2 C_{4}}
$$

and (2.1) obviously holds if $C_{3} \geqslant \exp \left(2 C_{4}\right)$.

Case 2. $\sup _{t \in B_{p^{\prime}}^{n}}\left\|\sum_{i=1}^{n} t_{i} Z_{i}\right\|_{2} \leqslant 2 u<\sup _{t \in B_{p^{\prime}}^{n}}\left\|\sum_{i=1}^{n} t_{i} Z_{i}\right\|_{\infty}$. Let us then define

$$
q:=\sup \left\{r \geqslant 2 C_{4}: \sup _{t \in B_{p^{\prime}}^{n}}\left\|\sum_{i=1}^{n} t_{i} Z_{i}\right\|_{r / C_{4}} \leqslant 2 u\right\} .
$$

By (2.2) we have

$$
\sup _{t \in B_{p^{\prime}}^{n}} \mathbb{P}\left(\left|\sum_{i=1}^{n} t_{i} Z_{i}\right| \geqslant u\right) \geqslant e^{-q}
$$

By (1.2), Theorem 2.1, and Chebyshev's inequality we have

$$
\mathbb{P}\left(S \geqslant C_{5} p(\mathbb{E} S+u)\right) \leqslant \mathbb{P}\left(S \geqslant e\|S\|_{q}\right) \leqslant e^{-q}
$$

for $C_{5}$ large enough. Thus (2.1) holds in this case.

Case 3. $u>\sup _{t \in B_{p^{\prime}}^{n}}\left\|\sum_{i=1}^{n} t_{i} Z_{i}\right\|_{\infty}=\|S\|_{\infty}$. Then $\mathbb{P}(S \geqslant u)=0$ and (2.1) holds for any $C_{2} \geqslant 1$.

In the proof of Theorem 1.1 we will use Theorem 2.1 from [6], which is a version of the results provided by Guédon-Rudelson in [8], and by Guédon-Mendelson-PajorTomczak-Jaegerman in [7]. Below we give only a particular version of the theorem; the general result is stated in [6].

Theorem 2.3 ([6, Theorem 2.1]). Let $X_{1}, \ldots X_{m} \in \mathbb{R}^{n}$ be independent random vectors, and let $p, q \geqslant 2$. Define

$$
u:=\sup _{t \in B_{p^{\prime}}^{n}}\left(\sum_{i=1}^{m} \mathbb{E}\left|\left\langle X_{i}, t\right\rangle\right|^{q}\right)^{1 / q}
$$


Estimates of norms of log-concave random matrices with dependent entries

and

$$
v:=\left(p^{9} \log m \mathbb{E} \max _{1 \leqslant i \leqslant m}\left\|X_{i}\right\|_{p}^{q}\right)^{1 / q}
$$

Then

$$
\left[\mathbb{E} \sup _{t \in B_{p^{\prime}}^{n}}\left|\sum_{i=1}^{m}\left(\left|\left\langle X_{i}, t\right\rangle\right|^{q}-\mathbb{E}\left|\left\langle X_{i}, t\right\rangle\right|^{q}\right)\right|\right]^{1 / q} \leqslant C(\sqrt{u v}+v) \leqslant 2 C(u+v) .
$$

\section{Proof of Theorem 1.1}

The next two lemmas provide estimates of the quantities $u$ and $v$ appearing in Theorem 2.3.

Lemma 3.1. Assume that $p, q, X$, and $Y$ are as in Theorem 1.1. Then

$$
\left(\mathbb{E} \max _{1 \leqslant i \leqslant m}\left\|X_{i}\right\|_{p}^{q}\right)^{1 / q} \leqslant C(p, q)\left[\max _{1 \leqslant i \leqslant m}\left\|A_{i}\right\|_{p}+\log m \mathbb{E} \max _{\substack{1 \leqslant i \leqslant m \\ 1 \leqslant j \leqslant n}}\left|X_{i j}\right|\right],
$$

where $C(p, q)$ depends only on $p$ and $q$.

Lemma 3.2. Assume that $p, q, X$, and $Y$ are as in Theorem 1.1. Then

$$
\sup _{t \in B_{p^{\prime}}^{n}}\left(\sum_{i=1}^{m} \mathbb{E}\left|\left\langle X_{i}, t\right\rangle\right|^{q}\right)^{1 / q} \leqslant C_{1} q \max _{1 \leqslant j \leqslant n}\left\|A^{(j)}\right\|_{q} .
$$

In the proof of Lemma 3.1 we will also need the following estimate:

Lemma 3.3. Assume that $Z$ is an isotropic log-concave vector in $\mathbb{R}^{m}$. Then for all $1 \leqslant k \leqslant m$ and all $a \in \mathbb{R}^{m}$ we have

$$
\mathbb{E} \max _{1 \leqslant i \leqslant m}\left|a_{i} Z_{i}\right| \geqslant \frac{1}{C_{6}} \max _{k \leqslant m}\left(a_{k}^{*} \min _{i \leqslant m}\left\|Z_{i}\right\|_{\log (k+1)}\right),
$$

where $\left(a_{i}^{*}\right)_{i=1}^{m}$ denotes the non-increasing rearrangement of $\left(\left|a_{i}\right|\right)_{i=1}^{m}$.

In order to prove Theorem 1.1, we repeat the scheme of the proof from [6].

Proof of Theorem 1.1. Let $u$ and $v$ be given by formulas (2.3) and (2.4). The triangle inequality, Theorem 2.3, Lemma 3.1, and Lemma 3.2 yield

$$
\begin{aligned}
\mathbb{E}\|X\|_{p^{\prime}, q} & \leqslant\left(\mathbb{E}\|X\|_{p^{\prime}, q}^{q}\right)^{1 / q}=\left[\mathbb{E} \sup _{t \in B_{p^{\prime}}^{n}} \sum_{i=1}^{m}\left|\left\langle t, X_{i}\right\rangle\right|^{q}\right]^{1 / q} \\
& \leqslant\left[\mathbb{E} \sup _{t \in B_{p^{\prime}}^{n}}\left|\sum_{i=1}^{m}\left(\left|\left\langle X_{i}, t\right\rangle\right|^{q}-\mathbb{E}\left|\left\langle X_{i}, t\right\rangle\right|^{q}\right)\right|\right]^{1 / q}+\sup _{t \in B_{p^{\prime}}^{n}}\left(\mathbb{E} \sum_{i=1}^{m}\left|\left\langle t, X_{i}\right\rangle\right|^{q}\right)^{1 / q} \\
& \leqslant C \cdot(u+v) \\
& \leqslant C(p, q)\left[(\log m)^{1 / q} \max _{1 \leqslant i \leqslant m}\left\|A_{i}\right\|_{p}+\max _{1 \leqslant j \leqslant n}\left\|A^{(j)}\right\|_{q}+(\log m)^{\frac{1}{q}+1} \mathbb{E} \max _{\substack{1 \leqslant i \leqslant m \\
1 \leqslant j \leqslant n}}\left|X_{i j}\right|\right] .
\end{aligned}
$$

The main contribution of this article lies in the proofs of Lemmas 3.1, 3.2, and 3.3.

Proof of Lemma 3.3. We may and do assume that $a_{1} \geqslant a_{2} \geqslant \ldots \geqslant a_{m} \geqslant 0$, i.e. $a_{i}^{*}=a_{i}$ for $i \leqslant m$. By [10, Proposition 3.3] we have for all $k \leqslant m$,

$$
\mathbb{E} \max _{1 \leqslant i \leqslant k}\left|a_{i} Z_{i}\right| \geqslant C^{-1} \min _{1 \leqslant i \leqslant k}\left\|a_{i} Z_{i}\right\|_{\log (k+1)} \geqslant C^{-1} a_{k} \min _{1 \leqslant i \leqslant m}\left\|Z_{i}\right\|_{\log (k+1)} .
$$

Thus

$$
\mathbb{E} \max _{1 \leqslant i \leqslant m}\left|a_{i} Z_{i}\right|=\max _{1 \leqslant k \leqslant m} \mathbb{E} \max _{1 \leqslant i \leqslant k}\left|a_{i} Z_{i}\right| \geqslant C^{-1} \max _{1 \leqslant k \leqslant m}\left(a_{k} \min _{1 \leqslant i \leqslant m}\left\|Z_{i}\right\|_{\log (k+1)}\right) .
$$


Estimates of norms of log-concave random matrices with dependent entries

Proof of Lemma 3.1. We may and do assume that $m \geqslant 2$.

Since we may approximate $A_{i j}$ by nonzero numbers, we may and do assume that $A_{i j} \neq 0$ for all $i, j$. Let $C_{2}, C_{3}$ be the constants from (2.1), let $C_{6}$ be the constant from Lemma 3.3, and recall that $C_{1}$ is the constant from (1.2). We may assume that all these constants are greater than 1 .

Note that for any $a, b \in \mathbb{R}$ we have $a=(a-b)_{+}+a \wedge b$. Thus, by the triangle inequality,

$$
\begin{aligned}
& \left(\mathbb{E} \max _{1 \leqslant i \leqslant m}\left\|X_{i}\right\|_{p}^{q}\right)^{1 / q} \\
& \leqslant\left(\mathbb{E} \max _{1 \leqslant i \leqslant m}\left[\left(\left\|X_{i}\right\|_{p}-C_{2} p \mathbb{E}\left\|X_{i}\right\|_{p}\right)^{q} \mathbf{1}_{\left\{\left\|X_{i}\right\|_{p} \geqslant C_{2} p \mathbb{E}\left\|X_{i}\right\|_{p}\right\}}\right]\right)^{1 / q}+C_{2} p \max _{1 \leqslant i \leqslant m} \mathbb{E}\left\|X_{i}\right\|_{p} .
\end{aligned}
$$

Moreover, for every $1 \leqslant i \leqslant m$ we have by (1.2) and the isotropicity of $Y_{i}$, that

$$
\begin{aligned}
\mathbb{E}\left\|X_{i}\right\|_{p} & \leqslant\left(\sum_{j=1}^{n} \mathbb{E}\left|Y_{i j}\right|^{p}\left|A_{i j}\right|^{p}\right)^{1 / p} \leqslant \max _{j \leqslant n}\left\|Y_{i j}\right\|_{p}\left\|A_{i}\right\|_{p} \leqslant C_{1} p\left\|A_{i}\right\|_{p} \\
& \leqslant C_{1} p \max _{1 \leqslant k \leqslant m}\left\|A_{k}\right\|_{p} .
\end{aligned}
$$

Now it suffices to estimate the first term of (3.2). Let

$$
B:=C_{1}^{2} C_{6} \log (m+1) \mathbb{E} \max _{\substack{1 \leqslant i \leqslant m \\ 1 \leqslant j \leqslant n}}\left|X_{i j}\right| \quad \text { and } \quad \sigma:=\left(\max _{1 \leqslant i \leqslant m} \sigma_{p, X_{i}}(2)\right) \vee B .
$$

By (2.1) and the integration by parts we have

$$
\begin{aligned}
\mathbb{E}_{1 \leqslant i \leqslant m} \max _{1 \leqslant i} & {\left[\left(\left\|X_{i}\right\|_{p}-C_{2} p \mathbb{E}\left\|X_{i}\right\|_{p}\right)^{q} \mathbf{1}_{\left\{\left\|X_{i}\right\|_{p} \geqslant C_{2} p \mathbb{E}\left\|X_{i}\right\|_{p}\right\}}\right] } \\
& \leqslant\left(2 C_{2} p e \sigma\right)^{q}+\int_{2 C_{2} p e \sigma}^{\infty} q v^{q-1} \mathbb{P}\left(\max _{1 \leqslant i \leqslant m}\left(\left\|X_{i}\right\|_{p}-C_{2} p \mathbb{E}\left\|X_{i}\right\|_{p}\right) \geqslant v\right) d v \\
& \leqslant\left(2 C_{2} p e \sigma\right)^{q}+\sum_{i=1}^{m} \int_{2 C_{2} p e \sigma}^{\infty} q v^{q-1} \mathbb{P}\left(\left\|X_{i}\right\|_{p}-C_{2} p \mathbb{E}\left\|X_{i}\right\|_{p} \geqslant v\right) d v \\
& =\left(2 C_{2} p e \sigma\right)^{q}+\left(C_{2} p\right)^{q} \sum_{i=1}^{m} \int_{2 e \sigma}^{\infty} q u^{q-1} \mathbb{P}\left(\left\|X_{i}\right\|_{p}-C_{2} p \mathbb{E}\left\|X_{i}\right\|_{p} \geqslant C_{2} p u\right) d u \\
& \leqslant\left(2 C_{2} p e \sigma\right)^{q}+\left(C_{2} p\right)^{q} C_{3} \sum_{i=1}^{m} \int_{2 e \sigma}^{\infty} q u^{q-1} \sup _{\|t\|_{p^{\prime}} \leqslant 1} \mathbb{P}\left(\left|\sum_{j=1}^{n} t_{j} X_{i j}\right| \geqslant u\right) d u
\end{aligned}
$$

We want to estimate the function we integrate in (3.4). Fix $u \geqslant 2 e \sigma$. For $i$ such that $u \geqslant \sup _{\|t\|_{p^{\prime}} \leqslant 1}\left\|\sum_{j=1}^{n} t_{j} X_{i j}\right\|_{\infty}$ the function we integrate vanishes, so from now on we will consider only $i$ 's for which $u<\sup _{\|t\|_{p^{\prime}} \leqslant 1}\left\|\sum_{j=1}^{n} t_{j} X_{i j}\right\|_{\infty}$.

Note that if $1 \leqslant i \leqslant m$ and $\sup _{\|t\|_{p^{\prime}} \leqslant 1}\left\|\sum_{j=1}^{n} t_{j} X_{i j}\right\|_{\infty}>u \geqslant e \sigma \geqslant e \sigma_{p, X_{i}}(2)$, then

$$
r:=r(i):=\sup \left\{s \geqslant 2: \sigma_{p, X_{i}}(s) \leqslant u / e\right\} \in[2, \infty)
$$

and $\sigma_{p}, X_{i}(r)=u / e$. Therefore

$$
\sup _{\|t\|_{p^{\prime}} \leqslant 1} \mathbb{P}\left(\left|\sum_{j=1}^{n} t_{j} X_{i j}\right| \geqslant u\right) \leqslant \frac{\sup _{\|t\|_{p^{\prime}} \leqslant 1}\left\|\left\langle t, X_{i}\right\rangle\right\|_{r}^{r}}{u^{r}}=e^{-r}
$$


Estimates of norms of log-concave random matrices with dependent entries

Now we will estimate $r$ from below. For $t \geqslant 2$ let

$$
\varphi(t)=t \min _{1 \leqslant j \leqslant n}\left\|Y_{i j}\right\|_{t}
$$

Since $Y_{i}^{\prime} s$ are identically distributed, $\varphi$ does not depend on $i$. By (1.2), and the isotropicity of $Y$ we have

$$
\begin{aligned}
\sigma_{p, X_{i}}(t) & \leqslant \sigma_{2, X_{i}}(t) \leqslant C_{1} t \max _{|x| \leqslant 1}\left(\mathbb{E}\left(\sum_{j=1}^{n} A_{i j} Y_{i j} x_{j}\right)^{2}\right)^{1 / 2}=C_{1} t \max _{|x| \leqslant 1}\left(\sum_{j=1}^{n} A_{i j}^{2} x_{j}^{2}\right)^{1 / 2} \\
& =C_{1} t \max _{1 \leqslant j \leqslant n}\left|A_{i j}\right| \cdot\left\|Y_{i j}\right\|_{2} \leqslant C_{1} \varphi(t) \max _{1 \leqslant j \leqslant n}\left|A_{i j}\right| .
\end{aligned}
$$

Since we can permute the rows of $A$, we may and do assume that

$$
\max _{1 \leqslant j \leqslant n}\left|A_{1 j}\right| \geqslant \ldots \geqslant \max _{1 \leqslant j \leqslant n}\left|A_{m j}\right| .
$$

Let $j(i) \leqslant n$ be such an index that $\left|A_{i j(i)}\right|=\max _{1 \leqslant j \leqslant n}\left|A_{i j}\right|$. Lemma 3.3 applied to $Z_{i}=Y_{i j(i)}$ and the non-increasing sequence $a_{i}=\left|A_{i j(i)}\right|$ implies

$$
\mathbb{E} \max _{\substack{1 \leqslant i \leqslant m \\ 1 \leqslant j \leqslant n}}\left|X_{i j}\right| \geqslant \mathbb{E} \max _{1 \leqslant i \leqslant m}\left|A_{i j(i)} Y_{i j(i)}\right| \geqslant C_{6}^{-1}(\log (m+1))^{-1} \max _{1 \leqslant i \leqslant m}\left(\varphi(\log (i+1))\left|A_{i j(i)}\right|\right),
$$

so for all $i \leqslant m$ we have

$$
B \geqslant C_{1}^{2} \varphi(\log (i+1))\left|A_{i j(i)}\right|=C_{1}^{2} \varphi(\log (i+1)) \max _{1 \leqslant j \leqslant n}\left|A_{i j}\right| .
$$

Note that by (1.2) for all $r \geqslant \lambda \geqslant 2$ we have $\sigma_{p, X_{i}}(r / \lambda) \geqslant \sigma_{p, X_{i}}(r) /\left(C_{1} \lambda\right)$. Take $\lambda=$ $\sigma_{p, X_{i}}(r) / B=u /(B e) \geqslant 2$. Then by a calculation similar to the one above we get

$$
\frac{u}{e}=\sigma_{p, X_{i}}(r) \leqslant \frac{C_{1} r}{2} \max _{1 \leqslant j \leqslant n}\left|A_{i j}\right| \leqslant C_{1}^{2} r \max _{\substack{1 \leqslant i \leqslant m \\ 1 \leqslant j \leqslant n}}\left|A_{i j}\right| \mathbb{E}\left|Y_{i j}\right| \leqslant C_{1}^{2} r \mathbb{E} \max _{\substack{1 \leqslant i \leqslant m \\ 1 \leqslant j \leqslant n}}\left|X_{i j}\right| \leqslant B r,
$$

so indeed $r \geqslant \lambda \geqslant 2$.

Therefore for all $i \leqslant m$ we have

$$
\frac{B}{C_{1}}=\frac{1}{\lambda C_{1}} \sigma_{p, X_{i}}(r) \leqslant \sigma_{p, X_{i}}(r / \lambda) \stackrel{(3.6)}{\leqslant} C_{1} \varphi\left(\frac{r}{\lambda}\right) \max _{1 \leqslant j \leqslant n}\left|A_{i j}\right| \leqslant \frac{B \varphi\left(\frac{r}{\lambda}\right)}{C_{1} \varphi(\log (i+1))} .
$$

Since the function $\varphi$ is strictly increasing, the previous inequality yields $r \geqslant \lambda \log (i+1)$. This together with (3.5) implies that (recall that $\lambda=\frac{u}{B e} \geqslant 2$ )

$$
\sum_{i=1}^{m} \sup _{\|t\|_{p^{\prime}} \leqslant 1} \mathbb{P}\left(\left|\sum_{j=1}^{n} t_{j} X_{i j}\right| \geqslant u\right) \leqslant \sum_{i=1}^{m}(i+1)^{-\frac{u}{e B}} \leqslant 2^{-\frac{u}{e B}}+\int_{2}^{\infty} x^{-\frac{u}{e B}} d x \leqslant 3 \cdot 2^{-\frac{u}{e \sigma}} .
$$

Inequalities (3.4), (3.8), and the Stirling formula yield

$$
\begin{aligned}
\left(\mathbb{E}\left[\max _{1 \leqslant i \leqslant m}\left(\left\|X_{i}\right\|_{p}-C_{2} \mathbb{E}\left\|X_{i}\right\|_{p}\right)^{q} \mathbf{1}_{\left\{\left\|X_{i}\right\|_{p} \geqslant C_{2} \mathbb{E}\left\|X_{i}\right\|_{p}\right\}}\right]\right)^{1 / q} & \leqslant\left(\left(2 C_{2} p e \sigma\right)^{q}+3\left(C_{2} p\right)^{q} C_{3} \int_{0}^{\infty} q u^{q-1} 2^{-\frac{u}{e \sigma}} d u\right)^{1 / q} \\
& \leqslant\left(\left(2 C_{2} p e \sigma\right)^{q}+\left(C C_{2} p \sigma\right)^{q} C_{3} \int_{0}^{\infty} q v^{q-1} e^{-v} d v\right)^{1 / q} \\
& \leqslant C C_{2} C_{3}^{1 / q} \sigma p q .
\end{aligned}
$$


Estimates of norms of log-concave random matrices with dependent entries

If $B \geqslant \max _{1 \leqslant i \leqslant m} \sigma_{p, X_{i}}(2)$, then $\sigma=B=C \log (m+1) \mathbb{E} \max _{\substack{1 \leqslant i \leqslant m \\ 1 \leqslant j \leqslant n}}\left|X_{i j}\right|$ and the assertion follows by (3.2), (3.3), and (3.9). Otherwise, by (1.2) we get

$$
\sigma=\max _{1 \leqslant i \leqslant m} \sigma_{p, X_{i}}(2) \leqslant 2 C_{1} \max _{1 \leqslant i \leqslant m} \sigma_{p, X_{i}}(1) \leqslant 2 C_{1} \max _{1 \leqslant i \leqslant m} \mathbb{E}\left\|X_{i}\right\|_{p}
$$

where the second inequality holds since the weak first moment is bounded above by the strong first moment. This together with (3.2), (3.3), and (3.9) gives the assertion.

Proof of Lemma 3.2. Since $p \geqslant 2, B_{p^{\prime}} \subset B_{2}$. Thus we may and do assume $p=2$. By (1.2), the isotropicity of $Y$, and the Jensen inequality we have

$$
\begin{aligned}
\sup _{t \in B_{2}^{n}}\left(\sum_{i=1}^{m} \mathbb{E}\left|\left\langle X_{i}, t\right\rangle\right|^{q}\right)^{1 / q} & \leqslant C_{1} q \sup _{\|t\|_{2} \leqslant 1}\left(\sum_{i=1}^{m}\left(\mathbb{E}\left|\left\langle X_{i}, t\right\rangle\right|^{2}\right)^{q / 2}\right)^{1 / q} \\
& =C_{1} q \sup _{\|t\|_{2}=1}\left(\sum_{i=1}^{m}\left(\sum_{j=1}^{n} A_{i j}^{2} t_{j}^{2}\right)^{q / 2}\right)^{1 / q} \\
& \leqslant C_{1} q \sup _{\|t\|_{2}=1}\left(\sum_{i=1}^{m} \sum_{j=1}^{n}\left|A_{i j}\right|^{q} t_{j}^{2}\right)^{1 / q} \\
& =C_{1} q\left(\sup _{\|t\|_{2}=1} \sum_{j=1}^{n}\left\|A^{(j)}\right\|_{q}^{q} t_{j}^{2}\right)^{1 / q} \\
& =C_{1} q \max _{1 \leqslant j \leqslant n}\left\|A^{(j)}\right\|_{q}
\end{aligned}
$$

Remark 3.4. By the same reasoning as in the log-concave case, we may prove the following result for matrices with independent heavy tailed entries. Let $X$ be an $m \times n$ random matrix with entries $X_{i j}=A_{i j} Y_{i j}$, where $Y_{i j}$ are independent symmetric random variables such that $\mathbb{E} Y_{i j}^{2}=1$, and let $L>0$. Assume that for any $r \geqslant 2$ and any $1 \leqslant i \leqslant m$, $1 \leqslant j \leqslant n$ we have $\frac{r^{\beta}}{L} \leqslant\left\|Y_{i j}\right\|_{r} \leqslant L r^{\beta}$ with $\beta \in\left[\frac{1}{2}, 1\right]$. Then for every $p, q \geqslant 2$ we have

$$
\mathbb{E}\|X\|_{p^{\prime}, q} \leqslant C(p, q, L)\left[(\log m)^{1 / q} \max _{1 \leqslant i \leqslant m}\left\|A_{i}\right\|_{p}+\max _{1 \leqslant j \leqslant n}\left\|A^{(j)}\right\|_{q}+(\log m)^{1 / q} \mathbb{E} \max _{\substack{1 \leqslant i \leqslant m \\ 1 \leqslant j \leqslant n}}\left|X_{i j}\right|\right] .
$$

where $C(p, q, L)$ depends only on $p, q$, and $L$.

At the end of Section 4 we provide another result concerning this type of random matrices (see Corollary 4.5).

In the proof of (3.10) one uses [12, Corollary 1.3], [13, Theorem 2.1], and (3.11) (see below) instead of (2.1), Lemma 3.3, and (3.6), respectively. The only non-trivial part is proving the claim:

$$
\left\|\sum_{j=1}^{n} t_{j} Y_{i j}\right\|_{r} \leqslant C L r^{\beta}\left\|\sum_{j=1}^{n} t_{j} Y_{i j}\right\|_{2}=C L r^{\beta}\|t\|_{2}
$$

where $C$ is an absolute constant, and repeating the proof of Theorem 1.1. By (3.11) we get

$$
\sigma_{p, c Y_{i}}(q) \leqslant C L q^{\beta} \sup _{s \in B_{p *}^{n}} \sqrt{\sum_{j=1}^{n} s_{j}^{2} c_{j}^{2}}=C L q^{\beta} \max _{1 \leqslant j \leqslant n}\left|c_{j}\right| \leqslant C L^{2} \min _{j \leqslant n}\left\|Y_{i j}\right\|_{q} \max _{1 \leqslant j \leqslant n}\left|c_{j}\right|,
$$

which allows us to obtain a version of (3.6) for $\varphi(t):=\underset{\substack{1 \leqslant i \leqslant j \\ 1 \leqslant n}}{\min }\left\|Y_{i j}\right\|_{t}$. 
Estimates of norms of log-concave random matrices with dependent entries

Proof of (3.11). It suffices to consider $r=2 k$, where $k$ is an integer. Let $G=\left(G_{j}\right)_{j=1}^{n}$ be the standard $n$-dimensional Gaussian vector. Recall that for any $t \in \mathbb{R}^{n}$ and $r \geqslant 1$ we have $\left\|\sum_{j=1}^{n} t_{j} G_{j}\right\|_{r}=\|t\|_{2}\left\|G_{1}\right\|_{r} \sim\|t\|_{2} \sqrt{r}=\sqrt{r}\left\|\sum_{j=1}^{n} t_{j} Y_{i j}\right\|_{2}$.

By the assumptions on $Y_{i}$ and by the fact that $\beta \geqslant \frac{1}{2}$ we get

$$
\begin{aligned}
\left\|\sum_{j=1}^{n} t_{j} Y_{i j}\right\|_{2 k}^{2 k} & =\sum_{j_{1}+\ldots+j_{n}=k}\left(\begin{array}{c}
2 k \\
2 j_{1}, \ldots, 2 j_{n}
\end{array}\right) \mathbb{E} Y_{i 1}^{2 j_{1}} \ldots \mathbb{E} Y_{i n}^{2 j_{n}} t_{1}^{2 j_{1}} \cdots t_{n}^{2 j_{n}} \\
& \leqslant L^{2 k} \sum_{j_{1}+\ldots+j_{n}=k}\left(\begin{array}{c}
2 k \\
2 j_{1}, \ldots, 2 j_{n}
\end{array}\right)\left(2 j_{1}\right)^{2 j_{1} \beta} \cdots\left(2 j_{n}\right)^{2 j_{n} \beta} t_{1}^{2 j_{1}} \cdots t_{n}^{2 j_{n}} \\
& \leqslant(2 k)^{2 k \beta-k} L^{2 k} \sum_{j_{1}+\ldots+j_{n}=k}\left(\begin{array}{c}
2 k \\
2 j_{1}, \ldots, 2 j_{n}
\end{array}\right)\left(2 j_{1}\right)^{j_{1}} \cdots\left(2 j_{n}\right)^{j_{n}} t_{1}^{2 j_{1}} \cdots t_{n}^{2 j_{n}} \\
& \leqslant(2 k)^{2 k \beta-k}(C L)^{2 k} \sum_{j_{1}+\ldots+j_{n}=k}\left(\begin{array}{c}
2 k \\
2 j_{1}, \ldots, 2 j_{n}
\end{array}\right) \mathbb{E} G_{1}^{2 j_{1}} \ldots \mathbb{E} G_{n}^{2 j_{n}} t_{1}^{2 j_{1}} \cdots t_{n}^{2 j_{n}} \\
& =(2 k)^{2 k \beta-k}(C L)^{2 k}\left\|\sum_{j=1}^{n} t_{j} G_{j}\right\|_{2 k}^{2 k} \leqslant(2 k)^{2 k \beta}(C L)^{2 k}\left\|\sum_{j=1}^{n} t_{j} Y_{i j}\right\|_{2}^{2 k},
\end{aligned}
$$

which finishes the proof of (3.11).

\section{Estimates of norms of matrices in the case of Gaussian mix- tures}

Let us recall the definition of Gaussian mixtures from [5], where their significance is also described.

Definition 4.1. A random variable $X$ is called a (centred) Gaussian mixture if there exist a positive random variable $r$ and a standard Gaussian random variable $g$, independent of $r$, such that $X$ has the same distribution as $r g$.

We will work with matrices of the form $\left(R_{i j} B_{i j} G_{i j}\right)_{i \leqslant m, j \leqslant n}$ whose entries are Gaussian mixtures. We additionally assume that $R_{i j}=\left|Z_{i j}\right|^{\gamma}$, where $\gamma \geqslant 0$, and that the matrix $Z$ is log-concave and isotropic (considered as a random vector in $\mathbb{R}^{m n}$ ). It will be clear from the proof, that the corollary below is true also for another type of matrices: $\left(R_{i} B_{i j} G_{i j}\right)_{i \leqslant m, j \leqslant n}$, where $R_{i}=\left|Z_{i}\right|^{\gamma}$, and $\left(Z_{1}, \ldots, Z_{m}\right)$ is an arbitrary isotropic logconcave random vector.

Corollary 4.2. Let $m, n \geqslant 2$, let $\gamma \geqslant 0$, let $B=\left(B_{i j}\right)$ be a deterministic $m \times n$ matrix, and let $G=\left(G_{i j}\right)_{i \leqslant m, j \leqslant n}$ be a random matrix whose entries are i.i.d. standard Gaussian variables. Let $X_{i j}=\left|Z_{i j}\right|^{\gamma} B_{i j} G_{i j}$, where $Z=\left(Z_{i j}\right)_{i \leqslant m, j \leqslant n}$ is a log-concave and isotropic random matrix independent of $G$. Then for every $p, q \geqslant 2 \vee \frac{1}{\gamma}$ we have

$$
\begin{aligned}
\mathbb{E}\|X\|_{p^{\prime}, q} \leqslant C(p, q, \gamma)\left[(\log m)^{\frac{1}{q}+\gamma} \max _{1 \leqslant i \leqslant m}\left\|B_{i}\right\|_{p}+(\log n)^{\gamma} \max _{1 \leqslant j \leqslant n}\left\|B^{(j)}\right\|_{q}\right. & \\
& \left.+(\log m)^{1 / q} \mathbb{E} \max _{\substack{1 \leqslant i \leqslant m \\
1 \leqslant j \leqslant n}}\left|X_{i j}\right|\right] .
\end{aligned}
$$

Proof. Inequality (1.1) applied to $a_{i j}=\left|Z_{i j}\right|^{\gamma} B_{i j}$ yields

$$
\begin{array}{r}
\mathbb{E}\|X\|_{p^{\prime}, q} \leqslant C(p, q)\left[(\log m)^{1 / q} \mathbb{E} \max _{1 \leqslant i \leqslant m}\left\|\left(B_{i j}\left|Z_{i j}\right|^{\gamma}\right)_{j}\right\|_{p}+\mathbb{E} \max _{1 \leqslant j \leqslant n}\left\|\left(B_{i j}\left|Z_{i j}\right|^{\gamma}\right)_{i}\right\|_{q}\right. \\
\left.+(\log m)^{1 / q} \mathbb{E} \max _{\substack{1 \leqslant i \leqslant m \\
1 \leqslant j \leqslant n}}\left|X_{i j}\right|\right],
\end{array}
$$


Estimates of norms of log-concave random matrices with dependent entries

so it suffices to prove that

$$
\mathbb{E} \max _{1 \leqslant i \leqslant m}\left\|\left(B_{i j}\left|Z_{i j}\right|^{\gamma}\right)_{j}\right\|_{p} \leqslant C(p, \gamma)(\log m)^{\gamma} \max _{1 \leqslant i \leqslant m}\left\|B_{i}\right\|_{p}
$$

and

$$
\mathbb{E} \max _{1 \leqslant j \leqslant n}\left\|\left(B_{i j}\left|Z_{i j}\right|^{\gamma}\right)_{i}\right\|_{q} \leqslant C(q, \gamma)(\log n)^{\gamma} \max _{1 \leqslant j \leqslant n}\left\|B^{(j)}\right\|_{q}
$$

for $p \geqslant 1 \vee \frac{1}{\gamma}$. By the symmetry of assumptions we need only to show (4.1).

If $\gamma<1$, then

$$
\mathbb{E} \max _{1 \leqslant i \leqslant m}\left\|\left(B_{i j}\left|Z_{i j}\right|^{\gamma}\right)_{j}\right\|_{p}=\mathbb{E} \max _{1 \leqslant i \leqslant m}\left\|\left(\left|B_{i j}\right|^{1 / \gamma}\left|Z_{i j}\right|\right)_{j}\right\|_{p \gamma}^{\gamma} \leqslant\left(\mathbb{E} \max _{1 \leqslant i \leqslant m}\left\|\left(\left|B_{i j}\right|^{1 / \gamma}\left|Z_{i j}\right|\right)_{j}\right\|_{p \gamma}\right)^{\gamma},
$$

and

$$
\left\|\left|B_{i}\right|^{1 / \gamma}\right\|_{p \gamma}^{\gamma}=\left\|B_{i}\right\|_{p}
$$

so it suffices to consider only $\gamma \geqslant 1$ (we used here the assumption that $p \geqslant \frac{1}{\gamma}$ ).

Note that for any $u \geqslant 1$ we have

$$
\begin{aligned}
\mathbb{E} \max _{1 \leqslant i \leqslant m}\left\|\left(B_{i j}\left|Z_{i j}\right|^{\gamma}\right)_{j}\right\|_{p} & =\mathbb{E} \max _{1 \leqslant i \leqslant m}\left\|\left(\left|B_{i j}\right|^{1 / \gamma} Z_{i j}\right)_{j}\right\|_{p \gamma}^{\gamma} \\
& \leqslant\left(\mathbb{E} \max _{1 \leqslant i \leqslant m}\left\|\left(\left|B_{i j}\right|^{1 / \gamma} Z_{i j}\right)_{j}\right\|_{p \gamma}^{u \gamma}\right)^{1 / u} \\
& \leqslant\left(\mathbb{E} \sum_{i=1}^{m}\left\|\left(\left|B_{i j}\right|^{1 / \gamma} Z_{i j}\right)_{j}\right\|_{p \gamma}^{u \gamma}\right)^{1 / u} \\
& \leqslant m^{1 / u} \max _{1 \leqslant i \leqslant m}\left(\mathbb{E}\left\|\left(\left|B_{i j}\right|^{1 / \gamma} Z_{i j}\right)_{j}\right\|_{p \gamma}^{u \gamma}\right)^{1 / u} .
\end{aligned}
$$

Fix $i \leqslant m$. By Theorem 2.1 applied to $p=p \gamma, q=u \gamma$ (recall that $\gamma \geqslant 1$, so $u \gamma \geqslant 1$, $p \gamma \geqslant p \geqslant 2$ ), and $Z_{j}=\left|B_{i j}\right|^{1 / \gamma} Z_{i j}$ we have

$$
\begin{gathered}
(C p \gamma)^{-\gamma}\left(\mathbb{E}\left\|\left(\left|B_{i j}\right|^{1 / \gamma} Z_{i j}\right)_{j}\right\|_{p \gamma}^{u \gamma}\right)^{1 / u} \leqslant\left[\mathbb{E}\left\|\left(\left|B_{i j}\right|^{1 / \gamma} Z_{i j}\right)_{j}\right\|_{p \gamma}+\sup _{t \in B_{p^{\prime}}^{n}}\left\|\sum_{j=1}^{n}\left|B_{i j}\right|^{1 / \gamma} Z_{i j} t_{j}\right\|_{u \gamma}\right]^{\gamma} \\
\leqslant 2^{\gamma-1}\left[\mathbb{E}\left\|\left(\left|B_{i j}\right|^{1 / \gamma} Z_{i j}\right)_{j}\right\|_{p \gamma}^{\gamma}+\sup _{t \in B_{p^{\prime}}^{n}}\left\|\sum_{j=1}^{n}\left|B_{i j}\right|^{1 / \gamma} Z_{i j} t_{j}\right\|_{u \gamma}^{\gamma}\right]
\end{gathered}
$$

We use (1.2) and the assumption $\mathbb{E} Z_{i j}^{2}=1$ to estimate the first term in (4.3):

$$
\mathbb{E}\left(\sum_{j=1}^{n}\left|B_{i j}\right|^{p}\left|Z_{i j}\right|^{p \gamma}\right)^{1 / p} \leqslant\left(\sum_{j=1}^{n}\left|B_{i j}\right|^{p} \mathbb{E}\left|Z_{i j}\right|^{p \gamma}\right)^{1 / p} \leqslant\left(C_{1} p \gamma\right)^{\gamma}\left\|B_{i}\right\|_{p} .
$$

Recall that $B_{p^{\prime}}^{n} \subset B_{2}^{n}$. We again use (1.2) and the isotropicity of $Z_{i}$ to estimate the second term in (4.3):

$$
\begin{aligned}
\sup _{t \in B_{p^{\prime}}^{n}}\left\|\sum_{j=1}^{n}\left|B_{i j}\right|^{1 / \gamma} Z_{i j} t_{j}\right\|_{u \gamma}^{\gamma} & \leqslant\left(C_{1} u \gamma\right)^{\gamma} \sup _{t \in B_{2}^{n}}\left\|\sum_{j=1}^{n}\left|B_{i j}\right|^{1 / \gamma} Z_{i j} t_{j}\right\|_{2}^{\gamma} \\
& =\left(C_{1} u \gamma\right)^{\gamma} \sup _{t \in B_{2}^{n}}\left(\sum_{j=1}^{n}\left|B_{i j}\right|^{2 / \gamma} t_{j}^{2}\right)^{\gamma / 2} \\
& =\left(C_{1} u \gamma\right)^{\gamma} \max _{1 \leqslant j \leqslant n}\left|B_{i j}\right| \leqslant\left(C_{1} u \gamma\right)^{\gamma}\left\|B_{i}\right\|_{p} .
\end{aligned}
$$

We take $u=\log m$ and put together (4.2), (4.3), and (4.4) to get the assertion. 
Estimates of norms of log-concave random matrices with dependent entries

Remark 4.3. Using Theorem 1.1 instead of (1.1) in the proof above yields a slightly worse estimate:

$$
\begin{aligned}
\mathbb{E}\|X\|_{p^{\prime}, q} \leqslant C(p, q)\left[(\log m)^{\frac{1}{q}+\gamma} \max _{1 \leqslant i \leqslant m}\left\|B_{i}\right\|_{p}+(\log n)^{\gamma} \max _{1 \leqslant j \leqslant n}\left\|B^{(j)}\right\|_{q}\right. & \\
& \left.+(\log m)^{1+\frac{1}{q}} \mathbb{E} \max _{\substack{1 \leqslant i \leqslant m \\
1 \leqslant j \leqslant n}}\left|X_{i j}\right|\right] .
\end{aligned}
$$

Remark 4.4. It is clear from the proof of Corollary 4.2 that in the case $Z_{i j}=G_{i j}^{\prime}$, where $G_{i j}^{\prime}$ are i.i.d. standard Gaussian variables, inequality (4.1) may be slightly improved:

$$
\mathbb{E} \max _{1 \leqslant i \leqslant m}\left\|\left(B_{i j}\left|G_{i j}^{\prime}\right|^{\gamma}\right)_{j}\right\|_{p} \leqslant C(p, \gamma)(\log m)^{\gamma / 2} \max _{1 \leqslant i \leqslant m}\left\|B_{i}\right\|_{p}
$$

In order to obtain this improvement one should use $\left\|\left\langle t, G_{i}\right\rangle\right\|_{u \gamma} \lesssim \sqrt{u \gamma}\left\|\left\langle t, G_{i}\right\rangle\right\|_{2}$ instead of $\left\|\left\langle t, Z_{i}\right\rangle\right\|_{u \gamma} \lesssim u \gamma\left\|\left\langle t, Z_{i}\right\rangle\right\|_{2}$. Thus the assertion of Corollary 4.2 in the case $Z_{i j}=G_{i j}^{\prime}$ (where $G^{\prime}$ is independent of $G$ ) states that

$$
\begin{array}{r}
\mathbb{E}\|X\|_{p^{\prime}, q} \leqslant C(p, q, \gamma)\left[(\log m)^{\frac{1}{q}+\frac{\gamma}{2}} \max _{1 \leqslant i \leqslant m}\left\|B_{i}\right\|_{p}+(\log n)^{\gamma / 2} \max _{1 \leqslant j \leqslant n}\left\|B^{(j)}\right\|_{q}\right. \\
\left.+(\log m)^{1 / q} \mathbb{E} \max _{\substack{1 \leqslant i \leqslant m \\
1 \leqslant j \leqslant n}}\left|X_{i j}\right|\right]
\end{array}
$$

Proof of Proposition 1.5. We begin similarly as in the proof of (4.1) (in the case $\gamma=1$ ), but we estimate the second term on the right-hand side of (4.3) in a slightly different way, using (1.2):

$$
\sup _{t \in B_{p^{\prime}}^{n}}\left\|\sum_{j=1}^{n} B_{i j} Y_{i j} t_{j}\right\|_{u} \leqslant n^{1 / u} \sup _{t \in B_{\infty}^{n}}\left(\mathbb{E} \max _{1 \leqslant j \leqslant n}\left|t_{j} B_{i j} Y_{i j}\right|^{u}\right)^{1 / u} \leqslant n^{1 / u} C_{1} u \mathbb{E} \max _{1 \leqslant j \leqslant n}\left|B_{i j} Y_{i j}\right| .
$$

We take $u=\log (m \vee n)$ to get the assertion.

We may use the result concerning Gaussian mixtures to obtain an estimate similar to the one from Remark 3.4, valid for all $\beta \geqslant \frac{1}{2}$ (not only for $\beta \in\left[\frac{1}{2}, 1\right]$ ), but with a slightly worse constants than in Remark 3.4. The proof is based on the fact, that the variables $Y_{i j}$ satisfying the moment assumption from Remark 3.4 are comparable with certain Gaussian mixtures.

Corollary 4.5. Let $m, n \geqslant 2, \beta \geqslant \frac{1}{2}, L>0$, and let $X$ be an $m \times n$ random matrix with entries $X_{i j}=A_{i j} Y_{i j}$, where $Y_{i j}$ are independent symmetric random variables such that $\mathbb{E} Y_{i j}^{2}=1$. Assume that for any $r \geqslant 2$ and any $1 \leqslant i \leqslant m, 1 \leqslant j \leqslant n$ we have $\frac{r^{\beta}}{L} \leqslant\left\|Y_{i j}\right\|_{r} \leqslant L r^{\beta}$. Then for all $p, q \geqslant 2$,

$$
\begin{aligned}
\mathbb{E}\|X\|_{p^{\prime}, q} \leqslant C(p, q, L, \beta)\left[(\log m)^{\beta+\frac{1}{q}} \max _{1 \leqslant i \leqslant m}\left\|A_{i}\right\|_{p}\right. & +(\log n)^{\beta} \max _{1 \leqslant j \leqslant n}\left\|A^{(j)}\right\|_{q} \\
& \left.+(\log m)^{1 / q} \sqrt{\log (m n)} \mathbb{E} \max _{\substack{1 \leqslant i \leqslant m \\
1 \leqslant j \leqslant n}}\left|X_{i j}\right|\right] .
\end{aligned}
$$

Proof. Let $G_{i j}, G_{i j}^{\prime}, i \leqslant m, j \leqslant n$, be i.i.d. standard Gaussian variables. Let $\left(\varepsilon_{i j}\right)$ be i.i.d. symmetric Bernoulli random variables, independent of $G$ and $G^{\prime}$. Note that $Y_{i j}^{\prime}:=\left|G_{i j}\right|^{2 \beta} \varepsilon_{i j}$ satisfies $\frac{r^{\beta}}{L^{\prime}} \leqslant\left\|Y_{i j}^{\prime}\right\|_{r} \leqslant L^{\prime} r^{\beta}$ for all $r \geqslant 2$, with a universal constant $L^{\prime}$, 
Estimates of norms of log-concave random matrices with dependent entries

since $\left\|G_{i j}\right\|_{s} \sim \sqrt{s}$ for $s \geqslant 1$. Let $X^{\prime}=\left(X_{i j}\right)$ be the $m \times n$ random matrix with entries $X_{i j}^{\prime}=A_{i j} Y_{i j}^{\prime}$. By [14, Lemma 4.7] we know that

$$
\frac{1}{C\left(L, L^{\prime}, \beta\right)} \mathbb{E}\left\|X^{\prime}\right\| \leqslant \mathbb{E}\|X\| \leqslant C\left(L, L^{\prime}, \beta\right) \mathbb{E}\left\|X^{\prime}\right\|
$$

for any norm $\|\cdot\| \mid$ on $m \times n$ real matrices. In particular

$$
\mathbb{E}\|X\|_{p^{\prime}, q} \leqslant C(L, \beta) \mathbb{E}\left\|X^{\prime}\right\|_{p^{\prime}, q}, \quad \text { and } \quad \mathbb{E} \max _{\substack{1 \leqslant i \leqslant m \\ 1 \leqslant j \leqslant n}}\left|X_{i j}^{\prime}\right| \leqslant C(L, \beta) \mathbb{E} \max _{\substack{1 \leqslant i \leqslant m \\ 1 \leqslant j \leqslant n}}\left|X_{i j}\right| .
$$

Moreover, by $\mathbb{E}\left|G_{i j}\right|=\sqrt{2 / \pi}$, the Jensen inequality, and (4.7) applied with $\gamma=2 \beta$, we have

$$
\begin{aligned}
& \mathbb{E}\left\|\left(X_{i j}^{\prime}\right)\right\|_{p^{\prime}, q}=\mathbb{E}\left\|\left(\varepsilon_{i j} A_{i j}\left|G_{i j}^{\prime}\right|^{2 \beta}\right)\right\|_{p^{\prime}, q}=\sqrt{\frac{\pi}{2}} \mathbb{E}\left\|\left(\mathbb{E}\left|G_{i j}\right| \varepsilon_{i j} A_{i j}\left|G_{i j}^{\prime}\right|^{2 \beta}\right)\right\|_{p^{\prime}, q} \\
& \leqslant \sqrt{\frac{\pi}{2}} \mathbb{E}\left\|\left(\left|G_{i j}\right| \varepsilon_{i j} A_{i j}\left|G_{i j}^{\prime}\right|^{2 \beta}\right)\right\|_{p^{\prime}, q}=\sqrt{\frac{\pi}{2}} \mathbb{E}_{X} \mathbb{E}_{G}\left\|\left(A_{i j} G_{i j}\left|G_{i j}^{\prime}\right|^{2 \beta}\right)\right\|_{p^{\prime}, q} \\
& \leqslant C(p, q)\left((\log m)^{\beta+\frac{1}{q}} \max _{1 \leqslant i \leqslant m}\left\|A_{i}\right\|_{p}+(\log n)^{\beta} \max _{1 \leqslant j \leqslant n}\left\|A^{(j)}\right\|_{q}\right. \\
& \left.+(\log m)^{1 / q} \mathbb{E} \max _{\substack{1 \leqslant i \leqslant m \\
1 \leqslant j \leqslant n}}\left|A_{i j} G_{i j}\right| \cdot\left|G_{i j}^{\prime}\right|^{2 \beta}\right) \\
& \leqslant C(p, q)\left((\log m)^{\beta+\frac{1}{q}} \max _{1 \leqslant i \leqslant m}\left\|A_{i}\right\|_{p}+(\log n)^{\beta} \max _{1 \leqslant j \leqslant n}\left\|A^{(j)}\right\|_{q}\right. \\
& \left.+(\log m)^{1 / q} \mathbb{E} \max _{\substack{1 \leqslant i \leqslant m \\
1 \leqslant j \leqslant n}}\left|G_{i j}\right| \mathbb{E} \max _{\substack{1 \leqslant i \leqslant m \\
1 \leqslant j \leqslant n}}\left|X_{i j}^{\prime}\right|\right)
\end{aligned}
$$

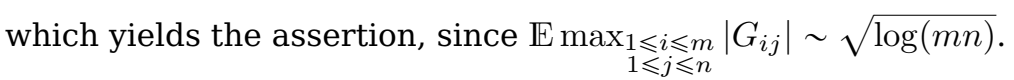

\section{The case of unconditional entries}

Proof of Corollary 1.6. Proceeding like in the proof of (1.4), we prove using (1.1) that

$$
\mathbb{E}\left\|\left(a_{i j} G_{i j}\right)\right\|_{p^{\prime}, q} \leqslant C(p, q)\left((\log m)^{1 / q} \mathbb{E} \max _{1 \leqslant i \leqslant m}\left\|\left(a_{i j} G_{i j}\right)_{j}\right\|_{p}+\mathbb{E} \max _{1 \leqslant j \leqslant n}\left\|\left(a_{i j} G_{i j}\right)_{i}\right\|_{q}\right) .
$$

where $G_{i j}$ are i.i.d. standard Gaussian variables.

Since $X$ is unconditional, it has the same distribution as the matrix $\left(\varepsilon_{i j} X_{i j}\right)_{i \leqslant m, j \leqslant n}$, where $\varepsilon_{i j}$ are i.i.d. symmetric Bernoulli variables independent of $X$. Let $G_{i j}$ be i.i.d. standard Gaussian variables independent of $X$ and $\left(\varepsilon_{i j}\right)_{i \leqslant m, j \leqslant n}$. Using $\mathbb{E}\left|G_{i j}\right|=\sqrt{2 / \pi}$, the Jensen inequality, and (5.1) (to estimate the mean with respect to $G$ ) we get

$$
\begin{aligned}
\mathbb{E}\left\|\left(X_{i j}\right)\right\|_{p^{\prime}, q} & =\mathbb{E}\left\|\left(\varepsilon_{i j} X_{i j}\right)\right\|_{p^{\prime}, q}=\sqrt{\frac{\pi}{2}} \mathbb{E}\left\|\left(\varepsilon_{i j} X_{i j} \mathbb{E}\left|G_{i j}\right|\right)\right\|_{p^{\prime}, q} \\
& \leqslant \sqrt{\frac{\pi}{2}} \mathbb{E}\left\|\left(\varepsilon_{i j} X_{i j}\left|G_{i j}\right|\right)\right\|_{p^{\prime}, q}=\sqrt{\frac{\pi}{2}} \mathbb{E}_{X} \mathbb{E}_{G}\left\|\left(X_{i j} G_{i j}\right)\right\|_{p^{\prime}, q} \\
& \leqslant C(p, q)\left((\log m)^{1 / q} \mathbb{E}_{X} \mathbb{E}_{G} \max _{1 \leqslant i \leqslant m}\left\|\left(X_{i j} G_{i j}\right)_{j}\right\|_{p}+\mathbb{E}_{X} \mathbb{E}_{G} \max _{1 \leqslant j \leqslant n}\left\|\left(X_{i j} G_{i j}\right)_{i}\right\|_{q}\right),
\end{aligned}
$$

We use (4.6) with $\gamma=1$ to get the assertion. 
Estimates of norms of log-concave random matrices with dependent entries

Remark 5.1. Using Theorem 1.1 instead of (1.1) in the proof above yields a slightly weaker estimate in Corollary 1.6 :

$$
\begin{aligned}
\mathbb{E}\|X\|_{p^{\prime}, q} \leqslant C(p, q)\left((\log m)^{\frac{3}{2}+\frac{1}{q}} \mathbb{E} \max _{1 \leqslant i \leqslant m}\left(\sum_{j=1}^{n}\left|X_{i j}\right|^{p}\right)^{1 / p}\right. & \\
& \left.+\sqrt{\log n} \mathbb{E} \max _{1 \leqslant j \leqslant n}\left(\sum_{i=1}^{m}\left|X_{i j}\right|^{q}\right)^{1 / q}\right) .
\end{aligned}
$$

\section{References}

[1] S. Artstein-Avidan, B. Klartag, and V. Milman, The Santaló point of a function, and a functional form of the Santaló inequality, Mathematika 51 (2004), no. 1-2, 33-48 (2005). MR-2220210

[2] G. Bennett, V. Goodman, and C. M. Newman, Norms of random matrices, Pacific J. Math. 59 (1975), no. 2, 359-365. MR-0393085

[3] C. Borell, Convex measures on locally convex spaces, Ark. Mat. 12 (1974), 239-252. MR0388475

[4] S. Brazitikos, A. Giannopoulos, P. Valettas, and B.H. Vritsiou, Geometry of isotropic convex bodies, Mathematical Surveys and Monographs, vol. 196, American Mathematical Society, Providence, RI, 2014. MR-3185453

[5] A. Eskenazis, P. Nayar, and T. Tkocz, Gaussian mixtures: entropy and geometric inequalities, Ann. Probab. 46 (2018), no. 5, 2908-2945. MR-3846841

[6] O. Guédon, A. Hinrichs, A.E. Litvak, and J. Prochno, On the expectation of operator norms of random matrices, Geometric aspects of functional analysis, Lecture Notes in Math., vol. 2169, Springer, Cham, 2017, pp. 151-162. MR-3645120

[7] O. Guédon, S. Mendelson, A. Pajor, and N. Tomczak-Jaegermann, Majorizing measures and proportional subsets of bounded orthonormal systems, Rev. Mat. Iberoam. 24 (2008), no. 3, 1075-1095. MR-2490210

[8] O. Guédon and M. Rudelson, $L_{p}$-moments of random vectors via majorizing measures, Adv. Math. 208 (2007), no. 2, 798-823. MR-2304336

[9] R. Latała, Some estimates of norms of random matrices, Proc. Amer. Math. Soc. 133 (2005), no. 5, 1273-1282. MR-2111932

[10] R. Latała, Sudakov-type minoration for log-concave vectors, Studia Math. 223 (2014), no. 3, 251-274. MR-3274967

[11] R. Latała and M. Strzelecka, Weak and strong moments of $\ell_{r}$-norms of log-concave vectors, Proc. Amer. Math. Soc. 144 (2016), no. 8, 3597-3608. MR-3503729

[12] R. Latała and M. Strzelecka, Comparison of weak and strong moments for vectors with independent coordinates, Mathematika 64 (2018), no. 1, 211-229. MR-3778221

[13] R. Latała and T. Tkocz, A note on suprema of canonical processes based on random variables with regular moments, Electron. J. Probab. 20 (2015), no. 36, 17. MR-3335827

[14] R. Latała, R. van Handel, and P. Youssef, The dimension-free structure of nonhomogeneous random matrices, Invent. Math. 214 (2018), no. 3, 1031-1080. MR-3878726

[15] S. Riemer and C. Schütt, On the expectation of the norm of random matrices with nonidentically distributed entries, Electron. J. Probab. 18 (2013), no. 29, 13. MR-3035757

[16] Y. Seginer, The expected norm of random matrices, Combin. Probab. Comput. 9 (2000), no. 2, 149-166. MR-1762786

Acknowledgments. I would like to thank Rafał Latała for suggestions which helped me to make the presentation clearer and more reader-friendly. 


\section{Electronic Journal of Probability Electronic Communications in Probability}

\section{Advantages of publishing in EJP-ECP}

- Very high standards

- Free for authors, free for readers

- Quick publication (no backlog)

- Secure publication $\left(\mathrm{LOCKSS}^{1}\right)$

- Easy interface (EJMS²)

\section{Economical model of EJP-ECP}

- Non profit, sponsored by $\mathrm{IMS}^{3}, \mathrm{BS}^{4}$, ProjectEuclid ${ }^{5}$

- Purely electronic

\section{Help keep the journal free and vigorous}

- Donate to the IMS open access fund ${ }^{6}$ (click here to donate!)

- Submit your best articles to EJP-ECP

- Choose EJP-ECP over for-profit journals

\footnotetext{
${ }^{1}$ LOCKSS: Lots of Copies Keep Stuff Safe http://www. lockss.org/

${ }^{2}$ EJMS: Electronic Journal Management System http://www.vtex.lt/en/ejms.html

${ }^{3}$ IMS: Institute of Mathematical Statistics http://www.imstat.org/

${ }^{4}$ BS: Bernoulli Society http://www. bernoulli-society.org/

${ }^{5}$ Project Euclid: https://projecteuclid.org/

${ }^{6}$ IMS Open Access Fund: http://www.imstat.org/publications/open.htm
} 